\title{
Biological and physical realizations of abstract metabolic models
}

\author{
ROBERT ROSEN ${ }^{1}$ \\ Committee on Mathematical Biology, \\ University of Chicago, Chicago, U.S.A.
}

EXTRAIT: Réalisations biologiques et physiques de modèles métaboliques abstraits. Il est possible de s'approcher de l'étude des actions métaboliques en considérant de modèles mathématiques formels qui incorporent les éléments essentiels du métabolisme. On peut développer ces modèles et déduire ses conséquences. Afn d'appliquer les résultats de ces études directement à la biologie, il faut remonter, partant du modèle formel, aux éléments individuels qui le réalisent. Pour l'ordinaire la classe du ces éléments est plus grosse, c'est à dire, elle contient plusieurs éléments sans signification biologique. En relation avec la transition, à partir du modèle formel, à une réalisation biologiquement intéressante, il se fait surgir de certains problèmes difficiles. Nous discutons ces problèmes et donnons l'idée de quelques techniques permettant d'exécuter les transitions indiquées. Nous appliquons celles-ci aux questions des systèmes analogues et de la simulation du métabolisme dans des systèmes non-biologiques.

\section{INTRODUCTION}

The study of biological systems can be approached from two distinct and complementary points of view. On the one hand, we can regard a biological system as nothing more than a complex aggregate of physico-chemical components and subsystems. Each of these components can, in principle, be separated from the others and studied in isolation by means of the standard techniques of physics and chemistry, either theoretical or experimental. The hope is that the properties of these physicochemical subsystems, when sufficiently well understood, will automatically provide an understanding of the typically biological activities of the system as a whole. The essence of this view, then, is to study a biological system by initially abstracting the biology out of the system and to investigate the remaining physico-chemical problems by means of familiar physico-chemical techniques. The biochemist and the molecular biologist have adopted this viewpoint towards biological systems with great success; the same holds true for the more classical subjects of mathematical biology (e. g., cell division, cardiovascular system, etc.). U.S.A.

${ }_{1}^{1}$ Present address: SUNY at Buffalo, Center for Theoretical Biology, Buffalo, N. Y., 
On the other hand, we can adopt a "Gestalt" or "System" approach to biological activity. Such an approach is concerned with the functional inter-relationships of the components of a complex biological system and is characteristically independent of the specific physico-chemical details of the structure of these components. General cybernetic approaches are of this type, as are the recent developments in mathematical biology, which have collectively been termed relational biology. Implicit in this point of view is the belief that a functional specification of the activities of a biological system, if properly done, will suffice to enable us to work backward to the details of the actual physical structures which must implement those functional activities.

Most of the difficult problems in biology arise from the fact that the purely structural and the purely functional approaches to biological activity do not, as yet, mesh very well. It remains most difficult to interpret structural data in functional terms, and vice versa; indeed, whenever such interpretations can be made, they serve to indicate how complex the structural basis of biologically meaningful functional activity can be. For instance, one of the striking aspects of BENZER's (1957) work on the fine structure of the bacteriophage chromosome was that three different functional interpretations of the functional term "gene", previously supposed equivalent, possessed different structural embodiments in that system.

The purpose of the present note is to indicate some techniques which may serve to bridge the gap between the functional and structural views of biological systems. These techniques belong to the realm of mathematical biology, which is unique in its ability to contribute both to the structural and the functional approaches to biological systems, and also to the relations between the two. It is hoped that such techniques will be important in relating work done on the abstract cybernetic properties of general regulatory systems to the specific structural problems which arise in the study of particular biological regulatory mechanisms.

\section{FUNCTIONAL MODELS AND THEIR REALIZATIONS}

Let us suppose that we are interested in the behavior of a particular kind of system. One way of studying this behavior is to make a mathematical model of the system, which may for example take the form of a differential equation, governing the manner in which certain variables of the system change with time. Once the model is constructed we turn from the study of the original system to a study of the model and its properties. For example, if the model takes the form of a differential equation, we can study the solutions of that equation under a variety of initial and boundary conditions, and we can study the properties of these solutions, such as stability,

However, implicit in the construction of such a model is a process of abstraction. As a result of this abstraction, we lose in general a unique specification of the system in which we were originally interested. That is, the mathematical model which we have constructed now applies not only to the original system, but to a large class of different systems. Once we have passed to the level of the mathematical model, then there is no way within the model to pass back to the original system. In particular, if we 
start with a mathematical model, as we always do in problems of functional organization, there is in general no way to specify individual systems which satisfy the model. At the level of the model, all such systems are indistinguishable, and each of them will be said to constitute a realization of the model.

A few examples may clarify this notion. Let us suppose we are given a differential equation of the form

$$
A \frac{d^{2} x}{d t^{2}}+B \frac{d x}{d t}+C x=D
$$

This equation represents a general oscillatory system. There is no way, within the context of the equation itself, to tell whether the equation refers to a mechanical oscillator, an electrical oscillator, or a hydrodynamic oscillator. Indeed, it is well known that if an equation of the form (1) can be realized mechanically, it can also be realized electrically and conversely; there exists an extensive literature on analogous systems of this type (OLSON 1958). This ambiguity lies at the root of the possibility of simulating one system by another; we shall have more to say about simulation as we proceed.

A second example, which we have discussed in detail elsewhere, and which may be of more direct biological interest, is the following. Much interest has been excited by the discovery of induction and repression mechanisms at the genetic level, and the control of gene expression by means of the operators described by JACOB \& MONOD (1961) and their co-workers. These authors have suggested a number of molecular mechanisms whereby these mechanisms could function in embryonic differentiation. It turns out that these mechanisms are formally identical to neural control circuits developed by LANDAHL (1941) to account for behavioral properties of organisms, such as learning and discrimination. These continuous models of LANDAHL are in turn equivalent to the discrete neural nets of McCulloch \& PitTs (1943). There is thus a precise sense in which these three diverse classes of systems are all realizations of the same abstract functional model and are hence analogous systems.

Of course, the ambiguity we have described at the level of the model itself, which is often called its generality, is one of the reasons that such models are so useful. Obviously, a wide variety of insights are obtainable merely from the study of such a general model in the abstract. But on the other hand, this very generality (which, we have seen, is to some degree a necessary property of such models) also lies at the heart of the difficulties encountered when we try to apply the results of a general system analysis to a particular biological system; for biologists are mainly interested in the properties of individual systems and not in diverse classes of such systems. Biologists must be able to identify the variables and parameters occurring in a general mathematical model with specifical structural (i. e., anatomical or biochemical) entities in each system with which they deal. Thus, in order to make general functional models meaningful and useful to the experimental biologist, it is necessary to be able to pass from the general model to that particular realization which corresponds to the specific biological system under investigation. 


\section{A DIRECT APPROACH: CONSTRAINTS}

Let us suppose at the outset that the class of physical systems which realize a particular abstract functional model is a well-defined entity. This supposition will suffice for the present discussion and will serve to bypass a host of difficult problems which we have discussed elsewhere (RosEN 1962). If this is granted, our problem becomes one of searching through this class to find specific realizations which are to be studied and compared with specific systems of interest.

Since initially, each model is obtained by a process of abstraction, a natural way of passing to individual realizations is to reverse the abstraction process. That is, we must now supplement the model by a variety of additional conditions, or constraints, which will be satisfied by some of the realizations of the original model, but not by others. The question now becomes: what sort of constraints, or supplementary conditions, are most suitable for the stipulation of biologically useful realizations of general models?

To illustrate the kinds of constraint which we shall propose, let us use a simple example from engineering. Let us consider an amplifying system, which functionally is specified by the condition that it multiply any input $F(t)$ by a number $k$, the amplification factor, to give an output $k F(t)$. We shall consider $F(t)$ to be an electrical signal varying in time (this already puts one constraint on the class of realizations).

The class of possible realizations of this functional system is diverse in several different ways. For one thing, it is known that an amplifier can be built on a variety of physical principles. Thus, we can build amplifiers which utilize vacuum tubes as operational components. Or we can build amplifiers which operate on solid-state principles (transistors). Or we can employ the principle of a resonating cavity, or the principle of the traveling-wave tube. A second source of diversity lies in the fact that, even if the physical basis of the amplification process is specified (another constraint on the class of realizations) an infinite variety of amplifier designs is still possible, simply in terms of circuitry. Finally, the physical lay-out of any one particular circuit can take an infinite variety of different forms.

Now we shall show how we may cut into these various forms of diversity by means of constraints which are meaningful in a biological context. The first constraint represents the specification of a particular structural embodiment of an aspect of the model. Thus, in the case of the amplifier, such a constraint would take the form (a): The operational components in the amplifier shall consist of a pair of 6L 6 triodes.

Once a constraint like (a) has been made a part of the model, a great deal of specific structural information can be inferred about those realizations which satisfy the constraint, simply on the basis of our knowledge of the physics of amplifiers and of the operation of 6L6 triodes. We know, for instance, that certain other specific circuit elements, such as resistors, inductances, capacitors and batteries, must be present.

We have succeeded, by imposing a constraint like (a), in cutting down the class of possible realizations of our amplifier system to a considerable extent. However, the class of realizations which satisfies (a) is still substantial. For instance, although we know that certain specific resistances must now occur in each of these realizations, we can say nothing about the physical embodiment of these resistances. Is it possible to 
introduce another constraint which, in combination. with the constraint (a), will furthur restrict the class of possible realizations?

We could, of course, add constraints of the form (a) for all the other circuit components. It seems much more appropriate, however, to supplement the single constraint (a) with one which will simultaneously imply such constraints over all the other circuitry. Such a constraint is (b): Our amplifier shall be such as to have the least cost of construction.

This constraint is an embodiment of the notion of "optimal design". Mathematically, what is involved here is the following: We are given a well-defined class of possible solutions to our problem; namely the class of all amplifiers satisfying the constraint (a). With each realization in the class we are able to associate a number, which in this case represents the cost of construction of that realization. If we minimize the cost over the class, then we may find ourselves left with one, or a small number, of our original realizations, which correspond to the minimum cost. We should note explicitly that the required optimality may well have reference to some other attribute of the system than the cost of construction; all that is required is a welldefined class of solutions and a function defined over that class which is to be minimized.

Considerations of optimal design are most natural to impose on biological systems, because optimality (i. e., minimality with respect to some kind of cost) is a consequence of the selection pressure exerted on all aspects of the structure and function of competing organisms. Indeed, the greater the selection pressure, the more rapid the convergence to the optimal structure. Notions of optimal design are implicit, at least qualitatively, in all aspects of theoretical biology, from the molecular to the ecological. We have discussed elsewhere in fuller detail the manner in which optimality principles can be applied rigorously to a wide variety of biological problems of the type we are discussing (Rosen 1966).

Let us sum up the results of the present section. We have argued that it is possible to pass from an abstract functional model directly to specific individual realizations of that model by imposing two constraints on the model: (a) choosing a specific physical embodiment of a particular functional subsystem, and (b) optimizing the remainder of the system around that specific physical embodiment, with respect to a suitably chosen notion of cost. In concrete terms, the implementation of these constraints is very similar to a paleontologist's reconstruction of an entire skeleton once a single bone has been supplied.

\section{INDIRECT APPROACH: ALTERNATE REALIZATIONS}

The advantage of the technique described in the preceding section is that it replaces conceptual problems by purely methodological ones. The methodological problems which remain may of course still be formidable; for instance, it is not always easy to decide what the appropriate cost constraint shall be. Consequently, it is often useful to take advantage of the ambiguity inherent in the original formal model, and 
the possibilities which arise for alternate realizations of the system in question. As we have mentioned above, such alternate realizations constitute analogous physical systems and open up wide possibilities for simulation.

Perhaps the best example of the manner in which the study of an alternate realization of a system of interest can shed light on the structural embodiments of the functional properties of the system comes from the work of STARK (1959) and his colleagues on the pupillary servomechanism. The simulation work carried out by STARK on this system revealed the presence of a definite number of lag elements in the pupillary control system. We cannot here dwell on the details of this work, except to make the point that as a result of this work it has been possible, at least in a preliminary way, to locate in the actual biological system the structural sites which manifest these lags. In the words of STARK, "These system equations have focused attention on the existence of physiological mechanisms which, although active in shaping pupil behavior, obviously have not been satisfactorily identified or studied". With the help of an alternate realization of the retinal system, $i$. e., by simulation, it has been possible to identify these mechanisms, and more, to obtain information as to the structural embodiment of these mechanisms in the actual biological system itself.

The study of alternate realizations of biological systems, especially realizations in the realm of engineering, has been assuming considerable technological importance. The disciplines of bionics (ROSEN 1963) and biomedical engineering are devoted almost exclusively to such studies. The recognition of an engineering system, say, as an alternate realization of some biological activity can be expected to throw light on the structural basis of the biological activity, as in the work of STARK just described. Although the direct approach sketched in the preceding section is perhaps more fundamental than is the study of alternate realizations, the difficulties implicit in the former may make the latter the more important tool, at least temporarily.

\section{SUMMARY}

1. The application of general models to individual biological systems requires us to be able to pass from the abstract model to individual elements of the class of realizations of the model.

2. In order to do this, it is necessary to supplement the model by a variety of constraints.

3. It is argued that two kinds of constraints are sufficient for this purpose: (a) a constraint specifying a particular physical embodiment of some subsystem of the formal model, and (b) a constraint specifying the optimality of the total system with respect to a suitably chosen criterion of cost.

4. The employment of analogous systems, or simulation, to obtain structural information about a particular realization is briefly discussed. 


\section{LITERATURE CITED}

BENZER, S., 1957. The elementary units of heredity. In: The chemical basis of heredity. Ed. by W. D. McElroy \& B. Glass. Johns Hopkins Univ. pr., Baltimore, 70-93.

JACOB, F. \& MoNoD, J., 1961. Teleonomic mechanisms in cellular metabolism, growth and differentiation. Cold Spring Harb. Symp. quant. Biol. 26, 389-401.

Landahl, H. D., 1941. Quoted from Rashevsky, N., 1958: 1, Chapt. $14 \mathrm{ff}$.

McCulloch, W. S. \& PrtTs, W., 1943. Quoted from Rashevskx, N., 1958: 1, Chapt. 19.

Olson, H. F., 1958. Dynamical analogies. Van Nostrand, Princeton, N. J., 278 pp.

Rashevsky, N., 1958. Mathematical biophysics. 3rd ed. Dover Publ., New York, Vol. 2, 1-462.

Rosen, R., 1962. Church's thesis and its relation to the concept of realizability in biology and physics. Bull. math. Biophys. 24, 375-393.

- 1963. Relational biology and bionics. IRE Trans. milit. Electron. 7, 160-162.

- 1966. Optimality principles in biology. Butterworths \& Co., London (in press).

STARK, L., 1959. Stability, oscillations and noise in the human pupil servomechanism. Proc. Inst, Radio Engrs 47, 1925-1939. 